\title{
Analysis of the Distribution Characteristics of Antibiotic Resistance Gene Pollution and Related Moving Factors in the Main Facility Agricultural Soils in Liaoning Province
}

\section{Yi Wu ( $1045181691 @ q q . c o m)$}

Shenyang University College of Environment https://orcid.org/0000-0001-9248-3430

\section{Xun Zhang}

Shenyang University College of Environment https://orcid.org/0000-0002-1748-1379

\section{Baiyu Wang}

Shenyang University College of Environment

\section{Ying Wang}

Institute of Applied Ecology Chinese Academy of Sciences

\section{Yuzhu Gao}

Shenyang University College of Environment

\section{Xiaoxue Chen}

Shenyang University College of Environment

\section{Research Article}

Keywords: vegetable field soil, antibiotic resistance genes, mobile genetic elements, quinolone antibiotics, the resistance mechanisms, distribution characteristics

Posted Date: September 24th, 2021

DOI: https://doi.org/10.21203/rs.3.rs-847741/v1

License: (c) (1) This work is licensed under a Creative Commons Attribution 4.0 International License. Read Full License 


\section{Abstract}

To understand the distribution characteristics of antibiotic resistance genes (ARGs) and mobile genetic elements (MGEs) in vegetable soil in the main facility agriculture distribution centres of Liaoning Province, 13 soil samples from seven sites including Chaoyang Yandu Xincheng, Zhuanghe, and Fushun were collected to detect the species and abundance of ARGs and MGEs, and analyse their correlation. The results showed that the ARGs were mainly tetracycline and macrolide antibiotic resistance genes, the transportable elements were transposases, and the cell protection and cell discharge pump was the main resistance mechanism. The detection rate of tetm-02, oprj, sul2, blaTEM, fox5, ermX, ermB, and ermF was $100 \%$. The detection rate of integron Int/-1 and transposable enzyme tnpA-01 was $100 \%$. The highest content of antibiotic resistance genes was found in Qiandangpu Village, Daying Town, and Zhuanghe City, followed by Wutun Village, Miaojia Village, Daying Town, and Dalian. Correlation analysis showed a correlation between the abundance of ARGs and the abundance of MGEs in agricultural soil. There was a significant positive correlation between the abundance of ARGs and integron $(P<0.05)$. The results of this study provide data support for the assessment of soil ARG pollution levels and the effective prevention and control of ARG transmission risk in the main protected vegetable fields in Liaoning Province.

\section{Background}

Antibiotics are primarily secondary metabolites or artificially synthesised analogues produced by microorganisms (including bacteria, fungi, and Actinomycetes) with anti-pathogen or other activities. Recently, the extensive abuse of veterinary antibiotics in the fields of excessive medical treatment and animal husbandry has contributed to the surge of antibiotic residues in the environment and the emergence of many drug-resistant bacteria. Antibiotic resistance genes (ARGs) in the soil primarily come from the "internal resistance" of bacteria in the environment, soil microorganisms, human or animal manure, etc., and the main way to enter the soil is animal manure fertilisation. Most antibiotics used to prevent or treat infections in humans or animals and to promote fast growth in livestock are only partially metabolised and subsequently excreted in the excrement (Martinez 2009). Human activities have a serious impact on the microbial community and ARGs (Yan, et al. 2020). Antibiotics have become the new cause for concern in environmental pollution. Increase in the number of antibiotics produced, the emergence of ARGs in human body, the effect of antibiotic treatment, and the stability of the ecological environment has caused serious damage. ARGs as a new type of environmental pollutant was formally defined in 2006 (Pruden, et al. 2006), and has attracted increasing attention from the public.

Manure from the aquaculture industry contains large amounts of antibiotic residues, and fertilising the soil with this manure induces soil microorganisms to produce a large number of resistance genes. ARGs migrate through horizontal gene transfer of mobile genetic elements (MGEs) such as plasmids, transposons, and integrons, leading to the spread of antibiotic-resistant bacteria. Horizontal transfer of ARGs is the transfer of mobile genetic factors through conjugation, transformation, transduction, etc. The mobile genetic factors are primarily integrons, plasmids, transposons, etc. Among them, the integron, as a 
gene-assisted capture system, is located on plasmids and chromosomes, and is also an important molecular element for the horizontal transfer of resistance genes (Yang, et al. 2013). Owing to the unique structure that can capture and integrate resistant genes, bacteria develop resistance or even multiresistance, such that integrons are considered to play an important role in the propagation of ARGs (Ruiz, et al. 2003). (Zhu, et al. 2019)analysed residual levels and spatial distribution characteristics of four types of quinolone antibiotics. The results showed that the detection rates of fluxacin, ciprofloxacin, and enfloxacin were $100 \%$, and that of ofloxacin was $98.6 \%$. Quinolone antibiotics were widely distributed, and the concentration of antibiotics was the highest in farmland soil along the middle reaches of the Fenhe River. Four types of quinolone antibiotics and one type of tetracycline antibiotic were detected in eel culture wastes, and the correlation between residual antibiotics and ARGs was not significant $(P \geq$ 0.05); however, the change in microbial community had a significant impact on the occurrence of environmental ARGs $(P<0.05)$ (Huang, et al. 2020). The results showed that the pollution of ARGs in eel culture waste is extremely serious, and the change of microbial community has a significant effect on environmental antibiotic resistance. Currently, there are numerous reports on the distribution characteristics of ARGs in soil. (Ji, et al. 2012) detected eight types of resistance genes in soils around breeding farms in Shanghai, and the ARGs with high abundance were sulfonamide ARGs SuIA and Sull. (Wu, et al. 2010) analysed soil around farms in Beijing, Tianjin, Jiaxing, and Zhejiang and found 15 tetracycline resistance genes, among which the most abundant ARGs were tet $M$, tet $O$, tetQ, tet $W$, and tet $T$. Quantitative polymerase chain reaction (PCR) was used to detect tetracycline and macrolide ARGs in manure application soil in Harbin, and it was found that tetM and tetW tetracycline ARGs and emrB and emrF genes were in high abundance (Peng, et al. 2019). (Liu, et al. 2021) showed that 17 strains of Escherichia coli showed varying resistance to $\beta$-lactams, aminoglycosides, tetracyclines, quinolones, and chloramphenicol; however, they were primarily resistant to $\beta$-lactams and tetracyclines. The detection rate of tetA resistance genes was relatively high, indicating overuse of cephalosporins and tetracycline. According to the results, the abundance of ARGs in both the farm and fertilised soil increased.

To study the distribution characteristics and correlation of ARGs and MGEs in facility soils in Liaoning Province. In this study, the distribution characteristics of ARGs and MGEs in agricultural soils were analysed in Panjin, Jinzhou, Chaoyang, Tieling, Xinmin, Shenyang, Fushun, Liaozhong, and Zhuanghe counties of Liaoning Province.

\section{Methods}

\subsection{Sampling sites and collection}

Soil samples were collected from 13 large-scale facility agriculture planting areas sites in Liaoning Province. Surface soil samples $(5-10 \mathrm{~cm})$ were collected using soil cores. A portion of each soil sample was passed through a $2 \mathrm{~mm}$ sieve and soil DNA was directly extracted simultaneously. The basic information on each sample is presented in Table 1.

\section{Table 1}


Basic information on soil samples.

\begin{tabular}{|c|c|c|c|c|}
\hline Number & Sample & $\begin{array}{l}\text { Vegetable } \\
\text { varieties }\end{array}$ & $\begin{array}{l}\text { Concentration(ng/ } \\
\mu \mathrm{L})\end{array}$ & $\begin{array}{l}\text { 16s } \\
\text { rRNA }\end{array}$ \\
\hline C1 & $\begin{array}{l}\text { Jiao Jia Ying Zi Village, Luochi Town, } \\
\text { Chaoyang County }\end{array}$ & eggplant & 39.20 & 14.25 \\
\hline $\mathrm{C} 2$ & $\begin{array}{l}\text { Jiao Jia Ying Zi Village, Luochi Town, } \\
\text { Chaoyang County }\end{array}$ & cantaloupe & 76.40 & 14.51 \\
\hline CS & $\begin{array}{l}\text { Ci Jia Zi Township, Chaoyang twelve } \\
\text { county }\end{array}$ & spinach & 73.40 & 14.32 \\
\hline D & $\begin{array}{l}\text { Wutun, Miaojia Village, Dayin Town, } \\
\text { Zhuanghe River }\end{array}$ & spinach & 94.40 & 14.13 \\
\hline $\mathrm{F}$ & Fushun Gaowan Town & eggplant & 85.40 & 14.20 \\
\hline J1 & Zhongan Town, Jinzhou North Town & eggplant & 12.40 & 14.50 \\
\hline $\mathrm{J} 2$ & Zhongan Town, Jinzhou North Town & cantaloupe & 11.20 & 14.68 \\
\hline P1 & Feijia Village, Xinglong Tai District, Panjin & rapeseed & 116.0 & 14.46 \\
\hline P2 & Feijia Village, Xinglong Tai District, Panjin & $\begin{array}{l}\text { Chinese } \\
\text { cabbage }\end{array}$ & 98.60 & 14.68 \\
\hline S & Shenbei Magang Township & $\begin{array}{l}\text { Chinese } \\
\text { cabbage }\end{array}$ & 75.20 & 14.34 \\
\hline $\mathrm{L}$ & Liujianfang, Liaozhong & rapeseed & 374.0 & 14.78 \\
\hline$x$ & $\begin{array}{l}\text { Qiandangpu Village, Xinmin Qiandangpu } \\
\text { Town }\end{array}$ & $\begin{array}{l}\text { Chinese } \\
\text { cabbage }\end{array}$ & 41.20 & 14.59 \\
\hline $\mathrm{T}$ & Fanhe New Area, Tieling & $\begin{array}{l}\text { Chinese } \\
\text { cabbage }\end{array}$ & 29.80 & 14.27 \\
\hline
\end{tabular}

\subsection{Extraction of DNA}

Fresh soil $(0.5 \mathrm{~g})$ was used for DNA extraction, which was performed using the E.Z.N.A. ${ }^{\circledR}$ Soil DNA Kit (Omega Bio-tek, Norcross, GA, USA) as per the manufacturer's instructions. The purity and content of total DNA were measured using the NanoPhotometer ${ }^{\text {TM }}$ P-Class (Implen, Germany), and the A260/A280 was between 1.8 and 2.0, indicating the purity of DNA which was analysed later.

\subsection{Quantitative detection of high-throughput quantitative real-time PCR (HT-qPCR)}

ARG detection was completed using the Wafergen Smartchip ultra-high-throughput fluorescence quantitative PCR system. The system could run a $5184 \mathrm{~nm}$ upgraded qPCR each time. Seventy-two pairs of primers were set, including a 16s rRNA reference primer, eight transposable enzymes, two integrons, and 61 targeted ARGs, to quickly and accurately detect the diversity and abundance of ARGs in various 
environmental media. The PCR mixture was first added to the microporous chip using a nano-upgraded multi-sample sampler. Thereafter, qPCR was carried out on the cycler $(100 \mathrm{~mm}$ : 1× LightCycler 480 SYBR Green IMaster;500 nM of each primer囚2 ng/ $\mu \mathrm{L}$ DNA template).

The cycling program included denaturation at 95 for $10 \mathrm{~min}$. Thereafter, 40 cycles of amplification were performed $\left(95^{\circ} \mathrm{C}, 30 \mathrm{~s} ; 60^{\circ} \mathrm{C}, 30 \mathrm{~s}\right)$. The qPCR results were automatically analysed using the instrument's qPCR software. If $\mathrm{C}=31$ is set as the detection domain, two or more of the three replicates are detected, the deviation is $<20 \%$, and meets the curve fitting analysis, the detection is judged as the detection domain.

\subsection{Statistical analysis}

A cluster heat map was drawn using the $\mathrm{R}$ language package pheatmap (version: 1.0.8) to show the abundance distribution pattern of ARGs in different samples. The Circos graph can be drawn using the Perl Circos tool (version: 0.69-6) to visually present the types of ARG-resistant antibiotics detected in different samples. The R language Tukey HSD method was used to statistically analyse the difference in the abundance of ARGs in different samples. Origin drawing software was used to draw the column chart (Zhang, et al. 2020b). SPSS Pearson analysis was used to analyse the correlation between ARG migration, propagation, and diffusion and MGEs in the agricultural soil environment.

\section{Results}

\subsection{Distribution characteristics 3.1.1. Types of ARGs and MGEs}

Fifty-seven ARG subtypes and 10 MGEs were detected in different agricultural soils (Fig. 1). There is one type of aminoglycoside ARG (aac(6')-Ib(aka aacA4)-01); three types of multi-drug resistance genes (acrA03, acrA-05, acrF); 4 types of $\beta$-lactamase ARGs (ampC-04, blaSFO, blaTEM, fox5); four types of chloromycin ARGs (cm/A1-01, cmIA1-02, and $\mathrm{cm} x(A), \mathrm{cfr})$; 16 types of macrolide ARGs (erm(34), erm(35), erm(36), ermA, ermA/ermTR, ermB, ermC, ermF, ermK-01, ermK-02, ermT-01, ermT-02, ermX, ermY, oleC, and mefA); seven types of sulfonamide ARGs (sul1, sul2, dfrA1, dfrA12, sulA/folP-01, sulA/folP-03, and folA); 14 types of tetracycline ARGs (tet(34), tetA-02, tetG-01, tetG-02, tetL-02, tetM-01, tetM-02, tet0-01, tetPA, tetPB-01, tetPB-03, tetR-02, tetT, and tet $X$ ) and eight types of quinolone ARGs ( $q n r B, q n r D)$. The MGEs detected are two types of integrons (clntl-1(class1), intl-1(clinic)) and eight types of transposases (tnpA-01, tnpA-02, tnpA-03, tnpA-04, tnpA-05, tnpA-07, Tp614, IS613). The detection rate of tetM-02, oprJ, sul2, blaTEM, fox5, ermX, ermB, and ermF in samples was $100 \%$, and that of integron intt-1 and transposase tnpA-01 was $100 \%$.

Forty-two species of ARGs and eight species of MGEs were detected in the agricultural soil of Qiandangpu Village, Xinmin Qiandangpu Town. The species and number of ARGs and MGEs were the 
highest here. Twenty-nine ARGs and seven MGEs species were detected in agricultural soil in Jiaojiayingzi Village, Luochi Town, Chaoyang County, with the lowest number detected.

\subsubsection{Abundance analysis of ARGs and MGEs}

ARG composition and distribution characteristics of MGEs were different at different sampling points. To study the correlation between ARGs and MGEs, the concentration of ARGs and that of the internal reference gene 16s rRNA were normalised so as to reduce the deviation, and the relative abundance of each ARG was obtained. The correlation between major ARGs and MGEs at different sampling points was analysed. Comparative analysis by Heatmap showed that the ARGs with a higher average abundance in 13 agricultural soils were quinolone and $\beta$-lactamase antibiotic resistance genotypes. In addition, it showed that the distribution of ARGs in different soil sampling points and soils varied. The relative abundance of ARGs in sampling site in Wutun Miaojia Village in Daying Town of Zhuanghe was relatively high, with a value of 1.75. The relative abundance of ARGs in Gaowan Town of Fushun was low, with a value of 0.07 . The relative abundance of MGEs at the sampling site in Magang Township, north Shenyang, was high, with a value of 0.178 . The relative abundance of MGEs at the sampling site in Zhongan Town of Jinzhou Beizhen City was low, with a value of 0.012 (Fig. 2a). The relative abundance of ARGs was as follows: $D>T>P 2>C 2>L>C S>X>P 1>C 1>S>J 1>J 2>F$ (Fig. 2b). The relative abundance of MGEs was as follows: $S>T>C S>P 2>X>L>D>P 1>C 1>C 2>F>J 1>J 2$.

Through analysis, the distribution characteristics of ARGs and their corresponding MGEs of different vegetable varieties in the same agricultural soil also showed some differences. The relative abundance of ARGs in the sampling sites was as follows: $C 1$ (eggplant soil) < C2 (cantaloupe soil), $P 1$ (rapeseed soil) < $P 2$ (Chinese cabbage soil). The relative abundance of MGEs at the sampling points was as follows: $C 1>$ $C 2$ and $P 1<P 2$.

The relative abundance of quinolone ARGs was most closely related to the total soil resistance genes. The content of multiple drug resistance genes was low, indicating that multiple drug resistance genes did not play a dominant role in agricultural soil. The MGEs of the four agricultural soil sampling sites were dominated by transposases. Analysis of MGEs showed that the relative abundance of the $C 2$ integron in cantaloupe soil (0.011) was higher than that in eggplant soil (0.008), and the abundance of the $P 2$ integron in Chinese cabbage soil (0.019) was higher than that in rape soil (0.012). The relative abundance of transposase gene $C 1(0.0063)>C 2(0.0026), P 2(0.0059)>P 1(0.0028)$ in agricultural soil.

\subsection{Mechanism of soil resistance in agriculture}

By comparing and analysing the resistance mechanisms in different agricultural soils, we can clearly show the resistance mechanisms. The resistance mechanisms include cellular protection, efflux pump, antibiotic deactivation, transposase translocation and integrase (Fig. 3). Cell protection and cell effluent pump were the primary mechanisms of antibiotic resistance in the main facility agricultural soil sampling sites in Liaoning Province. Differences in resistance mechanisms among different sampling sites were 
noted. The number of resistance mechanisms detected in the sampling sites of Qiandangpu Village in Xinmin Qiandangpu Town was the highest. The proportion of resistance mechanisms of cell protection and extracellular discharge pump was the also the highest here, while the proportion of antibiotic inactivation was relatively low. The number of antibiotic resistance mechanisms detected in Jiaojiayingzi Village, Luochi Town, Chaoyang County was the least, and the proportion of resistance mechanisms of cell protection and extracellular efflux pump were low. The number of resistance mechanisms of transposase mobile elements detected in Jiaojiayingzi Village of Luochi Town, Chaoyang County was the smallest. The results showed that the resistance mechanism of different agricultural sampling sites had different characteristics.

\subsection{Correlation analysis of ARGs and MGEs}

To analyse the correlation between ARGs and MGEs their relative abundance was determined. There is a positive correlation between ARGs and MGEs $(P \geq 0.05)$ (Fig. $4 a)$. The relative abundance of ARGs was positively correlated with that of integrons $\left(P<0.05, R^{2}=0.286, Y=0.287 X+10.395\right)$ (Fig. 4b). The integron int $\mathrm{l}-1$ was positively correlated with ARGs $\left(P<0.05, R^{2}=0.325, Y=1.32 X+0.446\right)$ (Fig. 5). The results showed that integrons formed the main influencing factor of MGEs, and there was a significant positive correlation between integrons and ARGs.

\subsection{Correlation between the residual of quinolone antibiotics and quinolone ARGs}

A Pearson correlation was used to determine the correlation between quinolone antibiotics and their ARGs in soil. The results showed that the residual amount of quinolone antibiotics and the $r$ value and $P$ value of quinolone ARGs were 0.907 and 0.0465 , respectively. Therefore, there is a significant positive correlation between quinolone antibiotics and quinolone ARGs $(P<0.05,0.90<r<1)($ Table 2).

\section{Table 2}

Relationship between quinolone antibiotics and quinolone antibiotic resistance genes (ARGs).

\begin{tabular}{|lll|}
\hline & $\mathbf{r}$ & $\boldsymbol{P}$ \\
\hline $\mathrm{ARG}_{\mathrm{S}}$ & 0.907 & 0.0465 \\
\hline
\end{tabular}

\section{Discussion}

The proliferation and spread of ARGs pose a serious threat to human and livestock health. (Tang, et al. 2015) showed that after long-term application of organic fertiliser, the residual amount of tetracycline in soil was ramarkbally higher than that in soil without application of organic fertiliser. The problem of antibiotic resistance gene pollution in vegetable soil in Shenyang has been concerned (Wang, et al. 2021), 
and the problem of antibiotic resistance gene pollution in facility soil in Liaoning Province is even more serious. Long-term application of fertilisers containing antibiotics or genetic determinants cannot be ignored because of the accumulation and risk of antibiotics and arginine pose in paddy soil of southern China. Zhang Lanhe (Zhang, et al. 2016) showed that long-term application of organic fertiliser would increase the abundance of culturable drug-resistant bacteria in the soil and increase the detection rate of ARGs. The proportion of tetracycline resistant bacteria in greenhouse soil was approximately three times that in field soil. A large number of studies have shown that the fertility of greenhouse soil has changed due to excessive use of chemical fertilizers ( $\mathrm{Hu}$, et al. 2017). The length of the planting time will also have different effects on the greenhouse soil (Sun, et al. 2016). The results of the study show that for most soil fertility indicators, the impact of planting leafy vegetables on the soil is less than that of fruit vegetables (Wang, et al. 2020). Soil fertility, soil humidity and soil microbial community of soil in facility agriculture are all higher than that of farm soil. There are also certain effects on the antibiotic resistance genes.

This study indicated that cell protection and cell effluent pump were the main mechanisms of antibiotic resistance in the soil sampling sites of facility agriculture in Liaoning Province. For example, (Zhang, et al. 2020b) showed that the mechanism of ARGs in soils of different vegetable bases, and the main resistance mechanisms in soils were cellular efflux pump and antibiotic inactivation.

The distribution of ARGs and MGEs in different vegetable soils was different and had a certain influence on the abundance of ARGs and MGEs in soil. The reason leads to the differents between them is the different rhizosphere microecology of different vegetable varieties or genotypes and the different degradation of pollutants in rhizosphere soil, as well as the different absorption and accumulation of pollutants in soil by different vegetable varieties or genotypes, (Cai, et al. 2008). The results showed that there were significant differences in the absorption and degradation of phthalate esters in soil between different vegetable varieties owing to the differences in the content and composition characteristics of protein resistance genes (Tai, et al. 2012). Studies have shown that concentration of contaminated soil layer, $\mathrm{pH}$ of leaching solution, and leaching time significantly affect the migration behaviour of quinolones antibiotics norfloxacin, ciprofloxacin, lomefloxacin and enrofolxacin in soil $(P<0.05)$ (Zhang, et al. 2014,). Research shows that different crop farmland soil abundance of antibiotic resistance genes have significant difference $(P<0.05)$. Research shows that all types of resistance genes in different crop farmland soil dominates abundance is multi-resistant genes, the movable element in the soil on the abundance distribution of whole and its antibiotic resistance genes consistent abundance (Huang, et al. 2014). Du (Du, et al. 2020) has shown that the ARG pattern of farmland soil is primarily affected by farming practices. With the increase in the proportion of crop sown area, the diversity of ARGs increases with the increase in crop yield. The diversity of ARGs first decreases and then increases, and there is a ramarkable relationship between crop planting area and the diversity, abundance and composition of ARGs. Moreover, application of organic fertiliser can transfer a variety of resistance genes into the soil and increase the abundance and diversity of these resistance genes (Zhang, et al. 2020a). In the present study, the distribution characteristics of ARGs and MGEs were different in agricultural soils planted with different vegetable varieties. This may be different from the absorption and accumulation of ARGs in soil 
by different vegetable varieties, or different from the planting area of crops, leading to different content and composition of ARGs.

Quinolone antibiotics and quinolone antibiotic resistance genes in the soil had a significant positive correlation $(P<0.05)$. Antibiotics are responsible for the increased release of $A R G s$ into the environment (Tang, et al. 2015). (Blackwell, et al. 2009) has showed that the difference in the composition and content of quinolone compounds in two soil profiles is related to the physiological and biochemical characteristics of different plant roots and the different production methods of each farmer. For example, different farming or migration methods affect the activity and content of antibiotics in the soil. (Seyoum, et al. 2021) showed a positive correlation with corresponding antibiotics, tetracycline and oxytetracycline. Therefore, a positive correlation between the antibiotics and the ARGs was observed.

In the present study, a positive correlation was found between ARGs and MGEs, and a significant positive correlation between ARGs and integrons in the main facility agricultural soils in Liaoning Province was also noted $(P<0.05)$. The results showed that the integron primarily affected the mobile element. Studies have shown that the AGR was positively correlated with the integron $(P<0.05)$, and the integron Int $I-1$ was significantly positively correlated with the AGR $(P<0.05)$. Studies have shown that there are five types of integrons found at present, among which, class I integrons are the most common (Mazel 2006). Yan et al. (2020b) showed a highly significant positive correlation between the total gene copy number of MGEs and ARGs $(R=0.979, P<0.001)$, and a significant positive correlation between the total relative abundance of ARGs and MGEs $(R=0.882, P=0.009)$ in seven paddy soils (He, et al. 2020$)$. They showed that the antibiotic residue in greenhouse soil was higher than that in field soil, which might be related to the fact that the amount of fertiliser applied in greenhouse soil was greater than that in field soil. Longterm application of organic fertiliser would increase the abundance of culturable drug-resistant bacteria in the soil and increase the detection rate of ARGs. It was found that the resistance genes sul 2 and tetx were correlated with the concentrations of some homoantibiotic sulfonamides, and the abundance of ARGs was also generally correlated with the concentrations of non-homoantibiotic antibiotics (Zhang, et al. 2016). (Cheng, et al. 2019) showed that the higher abundance of MGEs in the soil of livestock and poultry farms with hilly areas of purple soil was significantly correlated with the abundance of ARGs $(\mathrm{P}<$ 0.05). MGEs may promote the migration and diffusion of ARGs in the soil of these livestock and poultry farms. These results are consistent with those of the present study, and could be attributed to the fact

that MGEs in the main facility agriculture soils in Liaoning Province are primarily integrons, and ARGs are positively correlated with the abundance of integrons.

\section{Conclusions}

This research focuses on the main facility agricultural soil of vegetable facilities, and the detection of field vegetable soil needs further study.

Through the detection and analysis of 13 agricultural soils from 10 sampling sites in Liaoning Province, 57 antibiotic resistance gene subtypes and 10 mobile elements were obtained. The distribution 
characteristics of antibiotic resistance genes and mobile elements were different in different agricultural soils. The resistance mechanisms of agricultural soils at the sampling sites were primarily cell protection, extracellular pumping, and antibiotic inactivation. The relative abundance of ARGs significantly positively correlated with the relative abundance of integron $(P<0.05)$, the macrolides resistance gene ermB significantly positively correlated with the mobile element $(P<0.05)$, and the integron Int I-1 significantly positively correlated with the antibiotic resistance gene $(P<0.05)$. The residues of quinolone antibiotics in agricultural soil are closely related to ARGs. Moreover, there is a significant positive correlation between them.

This experiment studied the distribution characteristics and correlation of ARGs and MGEs in agricultural soils of major vegetable facilities in Liaoning Province. Not only is the pollution of antibiotic resistance in Shenyang city serious, but the risk of contamination of antibiotic resistance genes in Liaoning Province is also high. MGEs are ubiquitous in biological genes. ARGs will migrate with the MGEs, which can lead to serious risks.

\section{Declarations}

\section{Ethics approval and consent to participate}

Not applicable

\section{Consent for publication}

Not applicable

\section{Availability of data and material}

Authors can confirm that all relevant data are included in the article.

\section{Competing interests}

Any financial benefit that has been generated has been directly applied to my research. All reviews were written by myself.

\section{Funding}

This work was supported by the [Funding Agency \#1, Liaoning Provincial Natural Science Foundation of China] under Grant [number 2019 ZD-0555];

[Funding Agency \#2, National Natural Science Foundation of China,] under Grant [number 31971515]. 


\section{Authors' contributions}

Yi Wu: Data curation,Investigation, Writing- Original draft preparation.

Xun Zhang: Validation, Investigation, Writing - Review \& Editing.

Baiyu Wang: Formal analysis, Investigation, Visualization.

Ying Wang: Supervision, Visualization, Investigation.

Yuzhu Gao: Resources, Data curation, Investigation.

Xiaoxue Chen: Resources, Formal analysis, Investigation.

\section{Acknowledgments}

Not applicable

\section{References}

1. Blackwell, P. A., et al. 2009 Effects of agricultural conditions on the leaching behaviour of veterinary antibiotics in soils. Chemosphere 75(1):13-19.

2. Cai, Q. Y., et al. 2008 Potential of Ipomoea aquatica cultivars in phytoremediation of soils contaminated with di-n-butyl phthalate. Environmental and Experimental Botany 62(3):205-211.

3. Cheng, Jianhua, Xiangyu Tang, and Chen Liu 2019 Characteristics of Antibiotic Resistance Genes in Various Livestock Feedlot Soils of the Hilly Purple Soil Region. Environmental Science 40(07):32573262.

4. Du, S., et al. 2020 Large-scale patterns of soil antibiotic resistome in Chinese croplands. Science of the Total Environment 712.

5. He, Yan, et al. 2020 Diversity of antibiotic resistance genes in paddy soils in Sichuan Province, China. Journal of Agro-Environment Science 39(06):1249-1258.

6. Hu, W. Y., et al. 2017 Soil environmental quality in greenhouse vegetable production systems in eastern China: Current status and management strategies. Chemosphere 170:183-195.

7. Huang, Fuyi, et al. 2014 Profiling of Antibiotic Resistance Genes in Different Croplands Environmental Science:1-9.

8. Huang, Wei, et al. 2020 The occurrence of antibiotic resistance genes in eel culture waste and its correlation with antibiotics and the microbial community Chinese Journal of Applied and Environmental Biology 26(05):1275-1281.

9. Ji, X. L., et al. 2012 Antibiotic resistance gene abundances associated with antibiotics and heavy metals in animal manures and agricultural soils adjacent to feedlots in Shanghai; China. Journal of 
Hazardous Materials 235:178-185.

10. Liu, C., et al. 2021 Distribution characteristics and potential risks of heavy metals and antimicrobial resistant Escherichia coli in dairy farm wastewater in Tai'an, China. Chemosphere 262.

11. Martinez, J. L. 2009 Environmental pollution by antibiotics and by antibiotic resistance determinants. Environmental Pollution 157(11):2893-2902.

12. Mazel, D. 2006 Integrons: agents of bacterial evolution. Nature Reviews Microbiology 4(8):608-620.

13. Peng, Jing, et al. 2019 Effects of chicken manure organic fertilizer on antibiotic resistance genes and integrase genes in soil. Chinese Journal of Environmental Engineering 13(04):984-991.

14. Pruden, A., et al. 2006 Antibiotic resistance genes as emerging contaminants: Studies in northern Colorado. Environmental Science \& Technology 40(23):7445-7450.

15. Ruiz, J., et al. 2003 Integron-mediated antibiotic multiresistance in Acinetobacter baumannii clinical isolates from Spain. Clinical Microbiology and Infection 9(9):907-911.

16. Seyoum, M. M., et al. 2021 Occurrence and distribution of antibiotics and corresponding antibiotic resistance genes in different soil types irrigated with treated wastewater. Science of the Total Environment 782.

17. Sun, J. T., et al. 2016 Contamination of phthalate esters, organochlorine pesticides and polybrominated diphenyl ethers in agricultural soils from the Yangtze River Delta of China. Science of the Total Environment 544:670-676.

18. Tai, Yiping, et al. 2012 Occurrence of Quinolone Antibiotics in the Soils from a Green and an Organic Vegetable Fields. Journal of Agro-Environment Science 31(1):125-130.

19. Tang, X. J., et al. 2015 Effects of long-term manure applications on the occurrence of antibiotics and antibiotic resistance genes (ARGs) in paddy soils: Evidence from four field experiments in south of China. Soil Biology \& Biochemistry 90:179-187.

20. Wang, Baiyu, et al. 2021 Distribution of typical antibiotic resistance genes and mobile genetic elements in vegetable

21. soils of Shenyang. Chinese Journal of Ecology:1-9.

22. Wang, Zhaofeng, Dianing Gong, and Yili Zhang 2020 Investigating the Effects of Greenhouse Vegetable Cultivation on Soil Fertility in Lhasa, Tibetan Plateau. Chinese Geographical Science 30(3):456-465.

23. Wu, N., et al. 2010 Abundance and Diversity of Tetracycline Resistance Genes in Soils Adjacent to Representative Swine Feedlots in China. Environmental Science \& Technology 44(18):6933-6939.

24. Yan, C. C., et al. 2020 Deciphering the toxic effects of metals in gold mining area: Microbial community tolerance mechanism and change of antibiotic resistance genes. Environmental Research 189.

25. Yang, Fengxia, et al. 2013 Impact factors of the accumulation, migration and spread of antibiotic resistance in the environment. Chinese Journal of Applied Ecology 24(10):2993-3002. 
26. Zhang, H. P., et al. 2020a Tracking resistomes, virulence genes, and bacterial pathogens in long-term manure-amended greenhouse soils. Journal of Hazardous Materials 396.

27. Zhang, Lanhe, et al. 2016 Pollution of Antibiotics Resistant Genes in Farmland Amended With Livestock Organic Manure Journal of Ecology and Rural Environment 32(04):664-669.

28. Zhang, Rufeng, et al. 2020b Distribution Characteristics of Antibiotic Resistance Genes and Mobile Genetic Elements in Beijing Vegetable Base Soils. Environmental Science 41(01):385-393.

29. Zhang, Xu, et al. 2014, Migration Behavior and Influence Factors of Quinolone Antibiotics in Soil. Journal of Agro-Environment Science 33(07),:1345-1350.

30. Zhu, Yuen, et al. 2019 Residual characteristics and risk assessment of quinolones in agricultural soil along the Fenhe River. Acta Scientiae Circumstantiae 39(06):1989-1998.

\section{Figures}

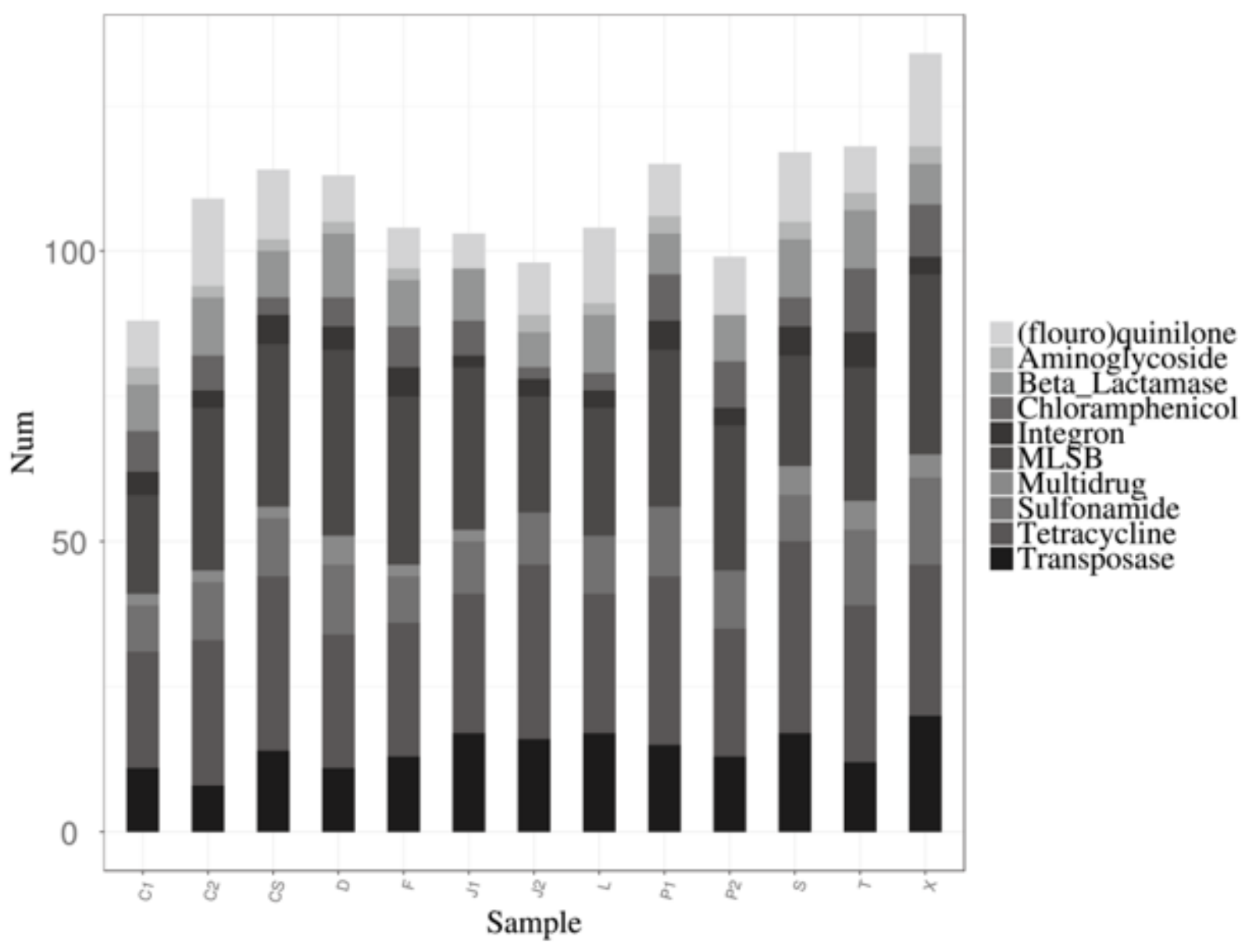

Figure 1

Distribution characteristics of antibiotic resistance genes and mobile genetic elements in agricultural soil of Liaoning Province. 


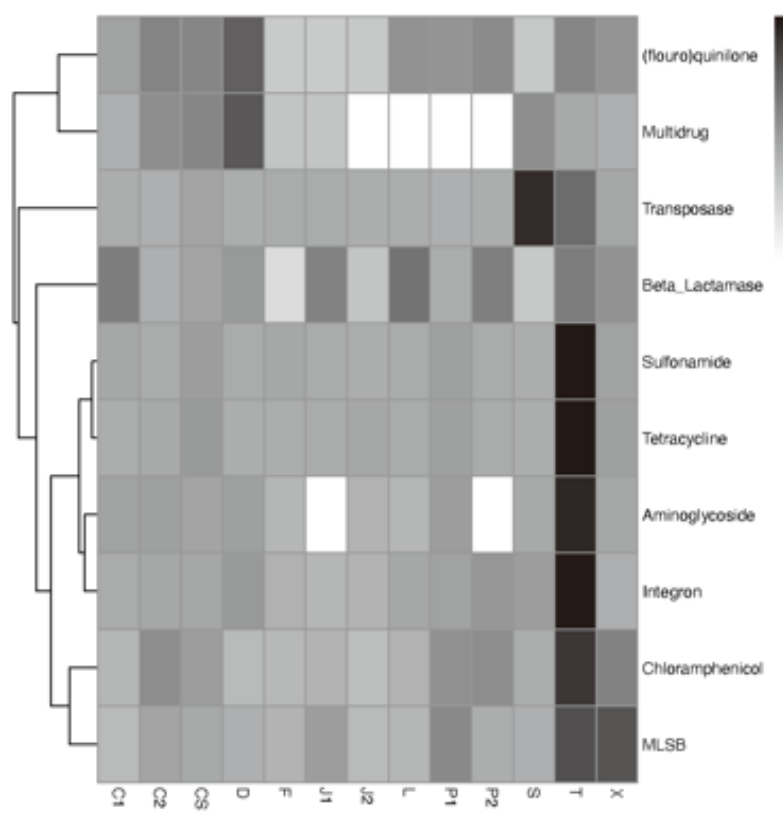

(a) Heat map of ARGs and MGEs

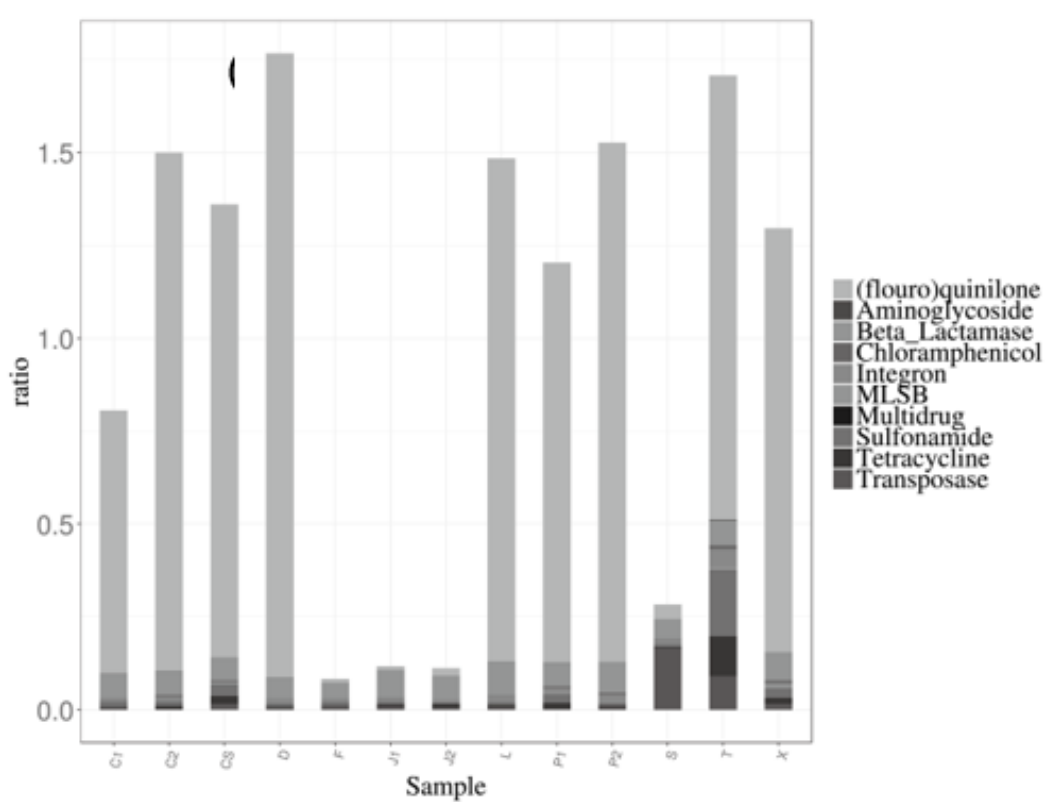

(b) The relative abundance of ARGs and MGEs

\section{Figure 2}

Comparative analysis of antibiotic resistance genotypes in different agricultural soils. 


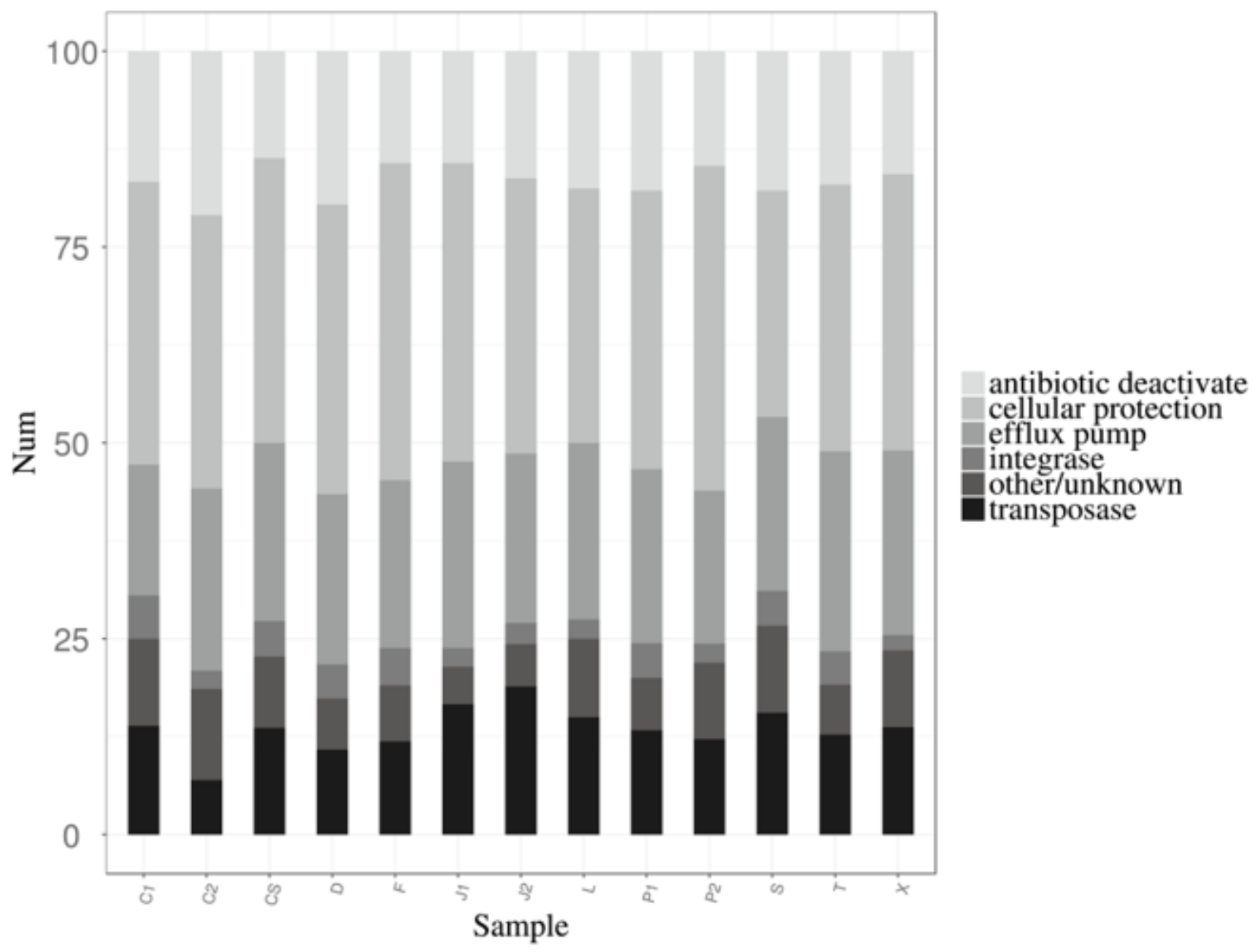

Figure 3

Mechanism analysis of soil resistance in agriculture. 
a

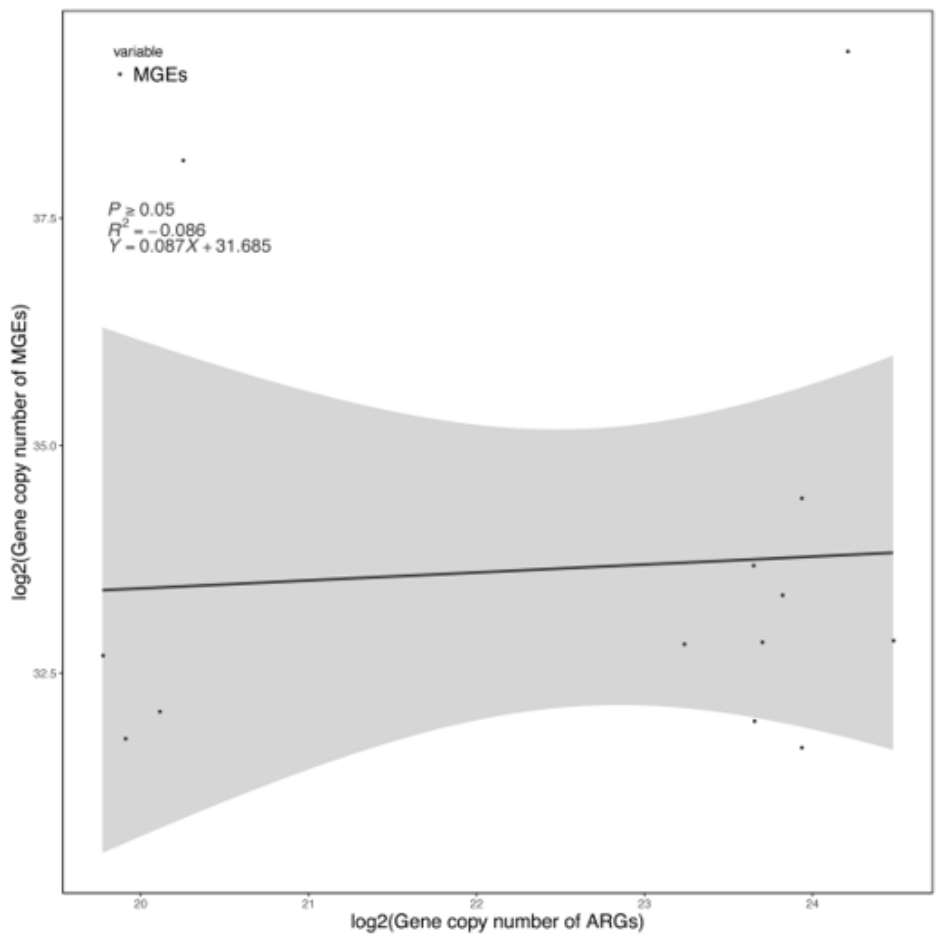

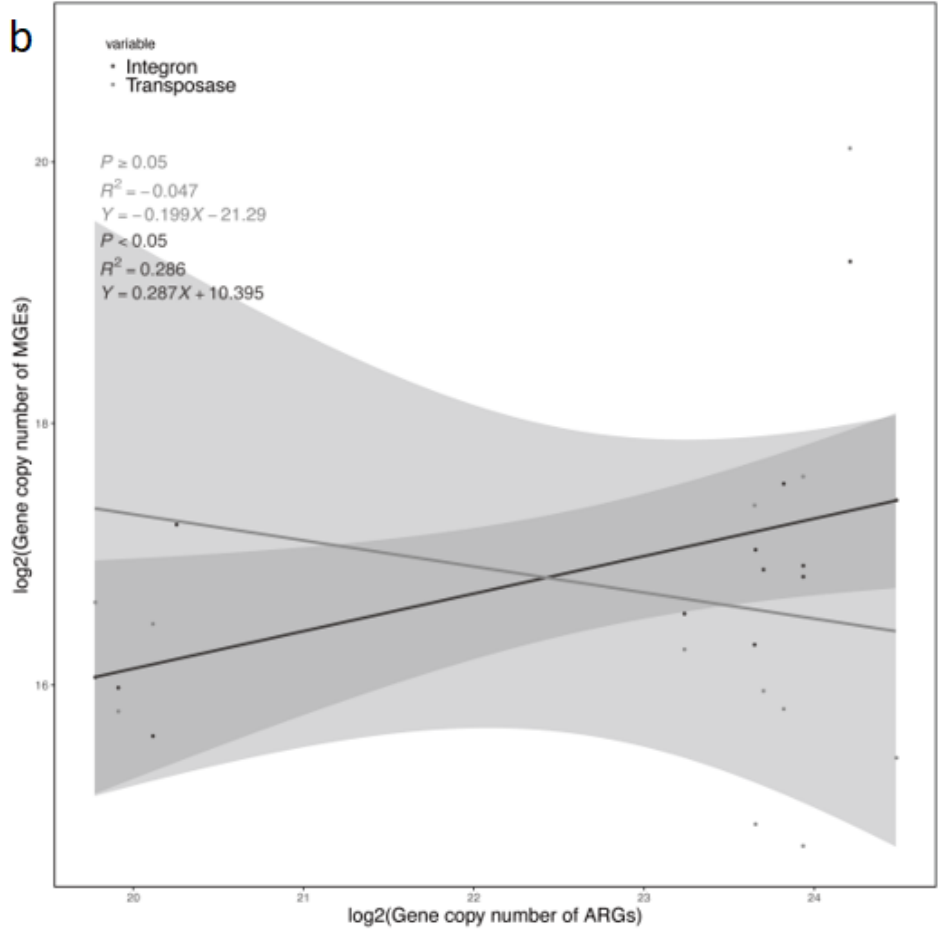

Figure 4

(a) Correlation analysis of antibiotic resistance genes (ARGs) and mobile genetic elements (MGEs) in agricultural soil of major facilities in Liaoning Province. (b) Correlation analysis between ARGs and integrator and mobile elements. 


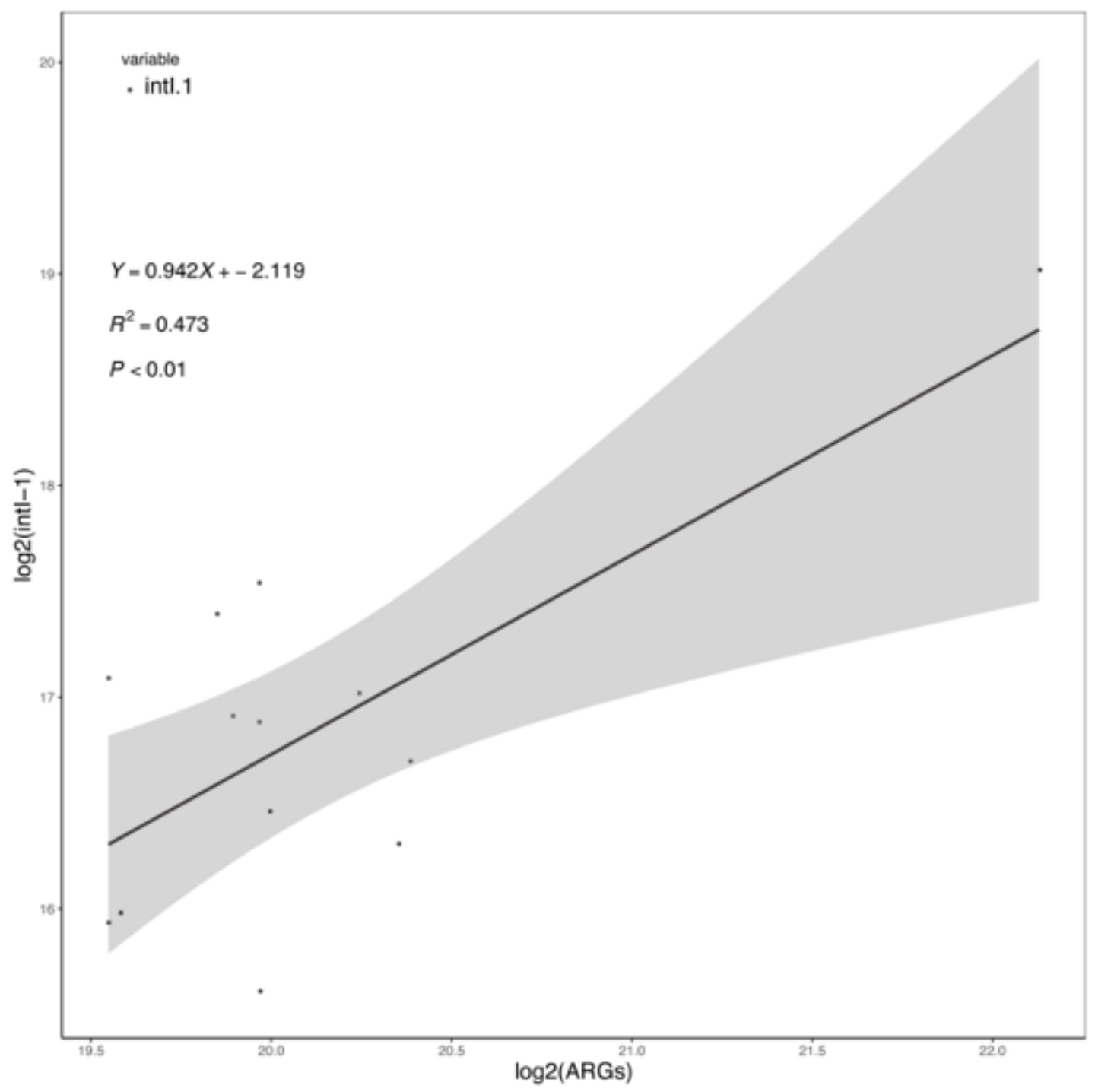

Figure 5

Correlation between antibiotic resistance genes and the intl-1 of movable element integrons.

\section{Supplementary Files}

This is a list of supplementary files associated with this preprint. Click to download.

- GA.png 